\title{
UV Imaging for In Vitro Dissolution and Release Studies: Initial Experiences
}

\author{
Jesper Østergaard ${ }^{1, *}$, Jim Lenke ${ }^{2}$, Sabrine S. Jensen ${ }^{1}$, Yu Sun ${ }^{1}$, \\ and Fengbin $\mathrm{Ye}^{1}$ \\ ${ }^{1}$ Department of Pharmacy, Faculty of Health and Medical Sciences, University of Copenhagen, Denmark \\ ${ }^{2}$ DissoFX, Maine, USA
}

\begin{abstract}
UV imaging has recently been introduced as a method in drug dissolution and release testing. Spatially and temporally resolved mapping of drug concentrations in a $7 \times 9 \mathrm{~mm}$ imaging area provides new opportunities for visualization and study of drug dissolution and release. This review describes the current instrumentation and principles of analysis. Pharmaceutical applications of UV imaging are presented, including determination of dissolution rates for drug substances, cocrystals, and nanocrystals and monitoring of solid form changes and single crystal dissolution as well as drug release and transport in hydrogels with relevance for characterization of parenteral depots. UV imaging may be of particular use when the amounts of material are sparse and detailed insights into dissolution and release processes are required, that is in solid form screening, preformulation, and early drug development.
\end{abstract}

KEYWORDS: Diffusion; dissolution; drug release; solid form changes; UV imaging.

\section{INTRODUCTION}

- or drug substances with poor aqueous solubility,

- dissolution frequently constitutes the rate-controlling step in oral drug absorption (1-7). Dissolution testing is conducted for several purposes in the pharmaceutical industry. One of these is to guide the drug development process including the selection of optimal solid forms, suitable excipients, and test formulations for further development. A continuing trend is to explore the physicochemical properties of the active substances by the use of high throughput techniques and to use the identified solid forms (salt, polymorphic, solvate, co-crystal, or amorphous) as a central part of the product development process $(6,8)$. Some of the most widely applied in vitro dissolution methods, including the rotating disc, paddle, and basket methods, require relatively large amounts of the active compound or formulation as well as dissolution medium. Consequently, in the early phases of drug development, miniaturized or micro-dissolution techniques needing only a few milligrams of the active pharmaceutical ingredient (API) are of particular interest and have been developed to overcome these practical limitations (9-14). Most dissolution testing methods rely on bulk solution concentration measurements as a function of time $(3,7)$ and may therefore suffer from delayed responses due to the need for accumulation of the solution concentration of the active. This may in turn make detection of, for instance, solid form changes during dissolution difficult $(6,15,16)$. Hence, real-time information during dissolution testing may be instrumental for understanding the complex behavior of an API or a formulation. This applies for drug substances or formulations intended for the oral route of administration as well as for

\footnotetext{
*Corresponding author.
}

parenterals, irrespective of the fact that several types of in vitro release testing devices and methods have been developed for parenteral formulations (17-21).

Imaging techniques providing spectrally, spatially, and temporally resolved information in real time are upcoming techniques in drug dissolution and release testing due to their high information content (22-30). Recently, UV imaging technology working in the transmission mode has become available, which may offer an alternative or complementary platform for conducting dissolution and release testing as compared with, for instance, Fourier transform infrared (FTIR) and magnetic resonance imaging (MRI). In UV imaging, the intensity of light at a selected wavelength in the UV range passing through a quartz cell is measured as a function of position and time (31). Since UV absorbance measurements are a suitable means for detection of many drug substances, UV imaging facilitates recording of temporally resolved concentration maps and quantification of drug substances in solution immediately adjacent to solid material (solid API or formulation).

We find that this comparatively simple form of chemical imaging holds significant potential in the areas of in vitro drug dissolution and release testing. The objective of the current work is to describe the instrumentation and basic principles of the technology to facilitate an appreciation of the advantages and limitations associated with UV imaging. Also, this review covers the current pharmaceutical applications of UV imaging in relation to dissolution and release studies.

\section{UV IMAGING TECHNOLOGY}

UV-vis spectrophotometry has for decades constituted one of the most important techniques for drug characterization and analysis. Chemical imaging is the construction of visual images of the distribution of analytes or 
components from the simultaneous measurement of spectral, spatial, and temporal information. Accordingly, UV imaging (and vis imaging) is the creation of images based on spatially and temporally resolved absorbance measurements. UV imaging can be performed in either reflectance or transmission mode utilizing commercially available instrumentation $(32,33)$. The present review is concerned with measurements performed in the transmission mode only, using the instrumentation developed by Paraytec Ltd. (York, UK) and now marketed by Sirius Analytical Instruments Ltd. (East Sussex, UK) as the Actipix SDI300 and currently as the Sirius SDI surface dissolution imaging system.

The commercially available UV imaging system consists of a low-volume flow cell integrated with a UV-vis camera as shown schematically in Figure 1 (31). The fluid containment of the cell consists of a PEEK sample holder sitting inside a parallel piped rectangular quartz tube, which constitutes the walls to both contain the dissolution medium and allow light to pass through the quartz cell (34). The sample holder is responsible for shaping the inlet and outlet flow across the sample surface to achieve laminar flow conditions. The heart of the technology is the UV camera paired with a broad-spectrum flash lamp providing the light to illuminate the $7 \times 9 \mathrm{~mm}(\mathrm{H} \times$ W) complementary metal oxide semiconductor (CMOS) camera chip (34). Using a band-pass filter, light with the wavelength of interest is selected and passed through the cell onto the CMOS camera chip (Figure 1). The light is transferred from the light source to the remote camera via a fiber-optic cable. The camera comprises 1.3 mega pixels $(1024 \times 1280, \mathrm{H} \times \mathrm{W})$ having a size of $7 \times 7 \mu \mathrm{m}$. Readout from the camera allows for individual pixel intensity val-

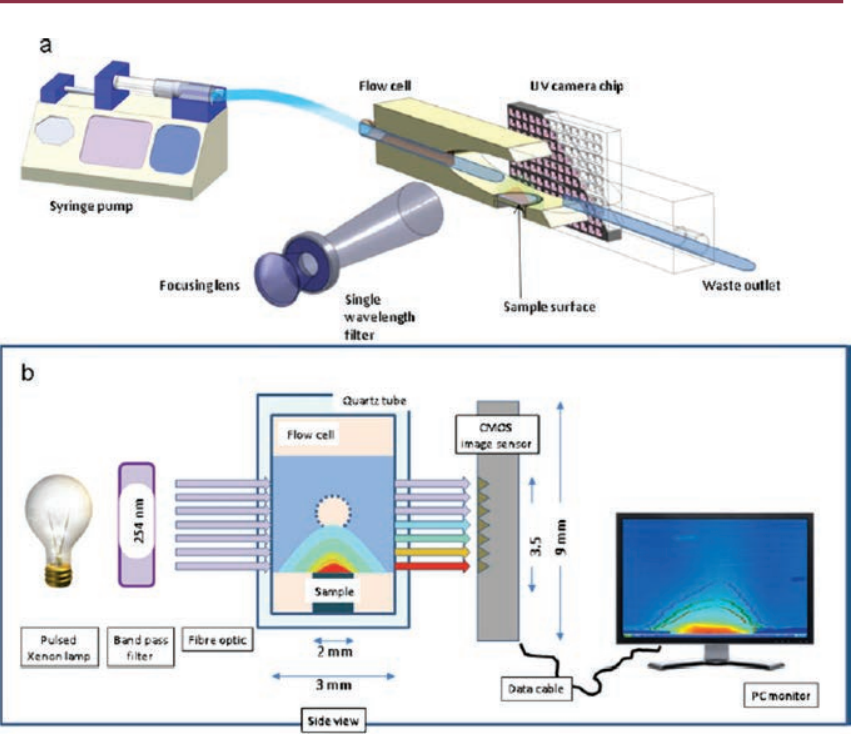

Figure 1. Schematic showing the setup of commercially available UV imaging instrumentation: (a) Key components of the instrument; (b) Side view of flow cell. Reprinted with permission from ref 31. Copyright 2010 Springer Science+Business Media. ues, and the pixels can be binned to improve sensitivity. Imaging rates are typically in the range of $0.5-8 \mathrm{~Hz}$. The recorded image files can be processed offline to provide absorbance values by subtracting both electronic noise and blank values obtained prior to sample insertion (34). The ability to detect at only one wavelength during a UV imaging experiment represents an obvious limitation of the instrumentation. This may make it difficult to interpret absorbance maps from dissolution and release experiments involving complex mixtures of UV-absorbing drug substances and excipients, for example. By applying one single wavelength, it is difficult to differentiate between absorbance, light scattering, and physical blocking of light (as will be pointed in the following sections). With respect to spatial resolution, the pixel size sets the limit, and a practical or functional characterization has not yet been published.

In the standard configuration (Figure 1), dissolution medium is pumped into the flow cell with the integrated syringe pump. The dissolution medium can be heated prior to entering the flow cell by passing the liquid through a temperature-controlled chamber to attain a temperature of $37^{\circ} \mathrm{C}$, for example, inside the cell. A stainless steel cylinder (2-mm inner diameter) containing the sample (3-10 $\mathrm{mg}$ ), usually a compressed drug substance, is positioned in the PEEK sample holder (Figure 1) allowing absorbance measurements at the compact surface $(31,34)$. The setup briefly outlined here is developed for flow-through type dissolution studies; however, the design of the camera housing allows for the integration with various types of sample holders and quartz cells.

\section{DRUG DISSOLUTION STUDIES \\ Flow-Through Dissolution Testing}

The Sirius SDI UV imaging system can be considered a miniaturized version of the flow-through device of Shah and Nelson (35) or a flow-through alternative to the miniaturized rotating disk intrinsic dissolution testing approaches $(9,10,12,34)$, that is, with some special opportunities due to the imaging functionality. It will only in special cases be able to hold an intact formulation, such as a tablet or capsule, and should not be compared to USP Apparatus 4 due to the small physical size.

The dissolution behavior of amorphous amlodipine besylate, amlodipine besylate anhydrate and dihydrate, and the free base of amlodipine (11) was investigated using the flow-through dissolution imaging setup described above. This first application of UV imaging for flow-through dissolution testing was performed at 355 $\mathrm{nm}$ with a flow rate of $0.2 \mathrm{~mL} / \mathrm{min}$. Approximately $6 \mathrm{mg}$ of the drug substance was compressed in the stainless steel cylinder (11). The UV images in Figure 2A show the dissolution of drug substance from the compacts as an increase in absorbance (shown with false color). Due to the presence of the flow, the drug is carried downstream from the position of the compact. The instrument software was 


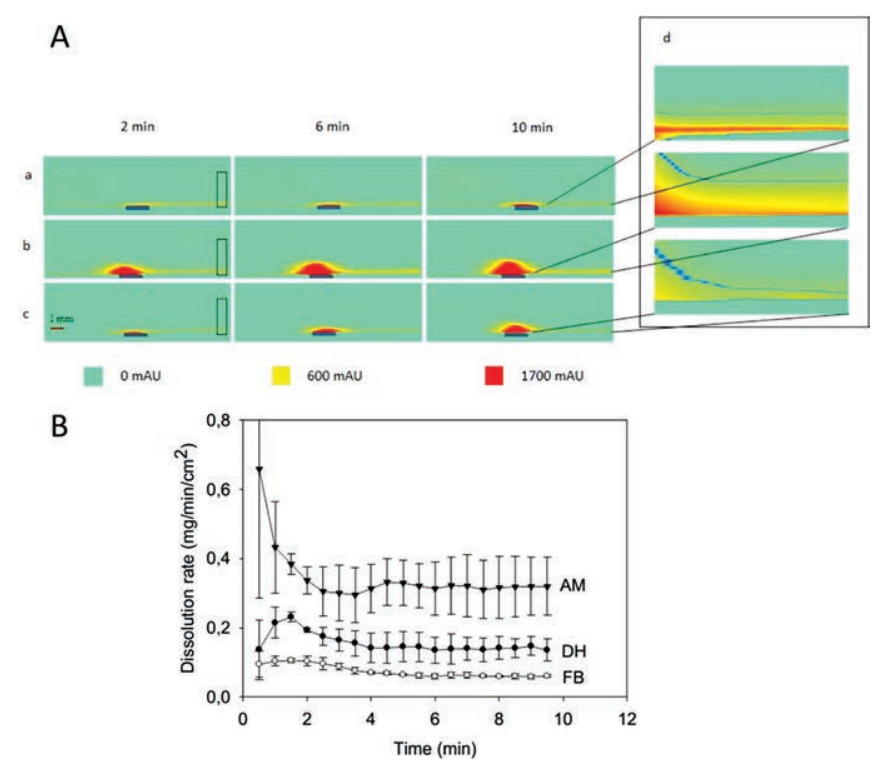

Figure 2. Amlodipine besylate dissolution behavior studied by UV imaging in acetate buffer, $\mathrm{pH}$ 4.5. (A) UV absorbance maps of dissolution of (a) amlodipine besylate dihydrate, (b) amorphous amlodipine besylate, and (c) amlodipine free base at $200 \mu \mathrm{L} / \mathrm{min}$. The inserts (d) show the spatially resolved concentration downstream from the sample compact. (B) Dissolution rates of amorphous amlodipine besylate (AM), amlodipine besylate dihydrate (DH), and amlodipine free base (FB) as a function of time determined by the UV imaging setup. The error bars represent the standard deviation $(n=3)$. Adapted with permission from ref 11. Copyright 2011 American Chemical Society.

used for calculating the dissolution rate using algorithms taking into account the flow rate and flow profile within the dissolution cell, the molar absorptivity of amlodipine besylate, and the surface area of the compact (11). Figure $2 \mathrm{~B}$ shows dissolution rate-versus-time profiles calculated based on the images. The dissolution rates for the three amlodipine forms at $10 \mathrm{~min}$ decrease in the order amorphous $>$ dihydrate $>$ free base. The absorbance maps for amorphous and free base samples indicate precipitation of solid material, swelling, or growth of crystals from the compact surface. UV absorbance measurements at a single wavelength do not allow distinguishing between absorbance or light scattering and blockage. However, visual inspection aided interpretation, and Raman spectroscopy verified that solid form changes occurred during dissolution. During dissolution, the amorphous amlodipine besylate transformed into the monohydrate form (11). This initial study highlighted that the combination of UV imaging and Raman spectroscopy can provide a deeper understanding of drug dissolution processes. UV imaging provided real-time information of early events occurring during dissolution. From the data presented on amlodipine besylate, it may be possible to estimate sensitivity and repeatability of the technique at the applied conditions. In general (with the exception of ref 31), there is a shortage of data related to the analytical performance of the UV imaging system.
Hulse et al. (36) applied the UV imaging-based flowthrough testing setup to investigate theophylline, indomethacin, and ibuprofen dissolution. The authors pointed to the importance of thorough solid form characterization prior to dissolution testing (e.g., by the use of XRPD and Raman spectroscopy). Theophylline anhydrate was observed to dissolve faster than theophylline monohydrate (36), which is consistent with the monohydrate being the stable form $(37,38)$. In contrast to a number of studies assessing the dissolution behavior of theophylline anhydrate $(15,37,39-41)$, indications of a solid form change during dissolution (in the form of a change in the IDR) was not observed. This was ascribed to differing hydrodynamic conditions inhibiting conversion to the hydrate form (36). Upon determination of dissolution rates, the authors found UV imaging sufficiently sensitive to discriminate between the dissolution performance of alpha and gamma indomethacin (36). UV imaging was also applied to micronized indomethacin material. Micronization did not affect the dissolution rates determined for gamma indomethacin samples, whereas for alpha indomethacin, the micronized material exhibited a lower dissolution rate than the unmicronized powder (36). The decrease was suggested to be from an increased exposure of hydrophobic surface domains upon micronization. Furthermore, the extensive study documented an increase in the rate of dissolution of sodium ibuprofen as compared with ibuprofen in phosphate buffer ( $\mathrm{pH}$ 7.2) and ibuprofen dissolution rate enhancement achieved through addition of sodium dodecyl sulfate to the dissolution medium (36).

The formation of cocrystals has attracted significant attention as a strategy to increase solubility and bioavailability of poorly soluble drug substances (42-45). In dissolution studies on carbamazepine, Li and co-workers $(46,47)$ expanded the application of UV imaging-based dissolution testing from single component/API studies to two-component systems. The dissolution of carbamazepine-nicotinamide cocrystals in $\mathrm{pH} 4.5$ acetate buffer was investigated and compared with the dissolution behavior of carbamazepine I, carbamazepine III, carbamazepine dihydrate, and a carbamazepine-nicotinamide physical mixture (47). Cautious selection of the detection wavelength allowed exclusive detection of carbamazepine (i.e., without significant contribution of nicotinamide to the UV absorbance). The dissolution rates for carbamazepine-nicotinamide cocrystal, carbamazepine I, carbamazepine II, and carbamazepine-nicotinamide physical mixture derived from the UV absorbance maps decreased as a function of time whereas the dissolution rate for the stable form carbamazepine dihydrate was constant over time. The majority of the UV imaging-based dissolution studies performed so far has emphasized the ability to follow the initial phases of the dissolution process $(11,36,48$, 49). However, the work of Li and coworkers (47) shows the ability to monitor dissolution processes over prolonged periods of time $(3 \mathrm{~h})$. The compacts of the carbamaze- 
pine-nicotinamide cocrystals exhibited increased dissolution rates during the entire period, whereas the dissolution rates of carbamazepine I, carbamazepine III, and the physical mixture were initially higher but decreased to the level of the dihydrate form within $2 \mathrm{~h}$ (47). Off-line Raman spectroscopy revealed that upon exposure to the dissolution medium, carbamazepine-nicotinamide cocrystal, carbamazepine I, carbamazepine III, and carbamazepinenicotinamide physical mixture transformed into the stable dihydrate form of carbamazepine. SEM images indicate a slower growth of the carbamazepine dihydrate crystal on the cocrystal compact surface as compared with the other solid forms tying up with the relatively higher dissolution rate sustained by the cocrystals (47). Recently, the effect of adding the surfactants sodium dodecyl sulfate and Tween 80 to the dissolution medium below and above the critical micelle concentration on the dissolution of carbamazepine-nicotinamide cocrystals, carbamazepine III, and carbamazepine-nicotinamide physical mixture was examined using UV imaging (46). Above the critical micelle concentration, SDS increased the dissolution of carbamazepine from the carbamazepine-nicotinamide cocrystal and carbamazepine III containing compacts, whereas, surprisingly, the presence of Tween 80 lead to a decrease in dissolution rate as compared with the dissolution rates determined in water. Solid-form characterization confirmed the occurrence of solution-mediated phase transformation into carbamazepine dihydrate during dissolution, allowing a discussion of the effect of the surfactants on crystal nucleation and extent of carbamazepine dihydrate crystallization during dissolution (46).

Boetker et al. (50) developed a numerical model simulating the hydrodynamics and the drug transport processes within the dissolution flow cell (CADISS-3) of the SDI300 UV imaging system. Laminar flow conditions existed in a wide flow rate range. The dissolution of paracetamol from compacts was investigated in the flow-through setup $(0.07-2.00 \mathrm{~mL} / \mathrm{min})$ applying UV imaging as well as quantification of paracetamol in the collected effluent by UV spectrophotometry for determination of the paracetamol dissolution rate. The ability of the finite element model to quantitatively predict the dissolution rate of paracetamol was established by comparison to the experimentally measured concentrations of paracetamol in the collected effluent. However, dissolution rates determined from the UV images were not in quantitative agreement with the results from the finite element simulations or the results derived from the effluent (50). The study points to the need for verifying the validity of dissolution rates determined from UV images by quantification of drug content in the effluent. The apparent error was suggested to be related to the angle of the incident light hitting the sensor or density gradients occurring during the dissolution of paracetamol (50). More work elucidating these aspects of UV imaging is clearly needed. Interestingly, in other studies $(48,51,52)$, better agreement between UV imaging and

collected effluent derived dissolution rates was observed (see below).

Recently, Østergaard et al. (52) successfully integrated the UV imaging flow-through setup with Raman spectroscopy allowing simultaneous solid-form characterization and dissolution monitoring. The proof-of-concept studies investigated the solvent-mediated phase transformations of theophylline anhydrate and sodium naproxen during dissolution into water and $0.1 \mathrm{M} \mathrm{HCl}$, respectively. Theophylline anhydrate transformed into the more stable monohydrate form within $\approx 5 \mathrm{~min}$ at the applied conditions (52), seemingly in contrast to the UV imaging study of Hulse et al. (36). However, this may stress the key feature of the combined UV imaging-Raman spectroscopic approach that solid form characterization is performed in situ (not offline) during dissolution testing on the very same material and at exactly the same hydrodynamic conditions, which could otherwise lead to different transformation kinetics.

\section{Dissolution Behavior in Biorelevant Media by UV Imaging}

The dissolution behavior of furosemide in biorelevant media containing $5 \mathrm{mM}$ sodium taurocholate and 1.15 $\mathrm{mM}$ phosphatidylcholine, $40 \mathrm{mM}$ sodium taurocholate and $10 \mathrm{mM}$ phosphatidylcholine, as well as the blank medium (phosphate buffer, $\mathrm{pH}$ 6.5) was investigated by UV imaging (48). UV imaging of furosemide dissolution was measured as a function of flow rate $(0.2-1.0 \mathrm{~mL} / \mathrm{min})$. The dissolution rate of furosemide decreased with a reduction of the flow rate. The dissolution rates obtained from the UV imaging show an overall quantitative agreement with those obtained from collected effluent samples.

Both methods indicate that the biorelevant media did not significantly increase the dissolution rate of furosemide. From the UV imaging maps (Figure 3 in ref 48), an area of intense absorbance is seen at the compact surface. To further investigate whether the upward shifts of the contour
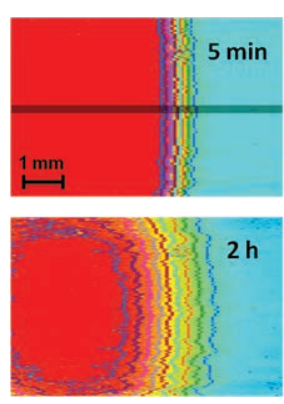

$2 \mathrm{~h}$

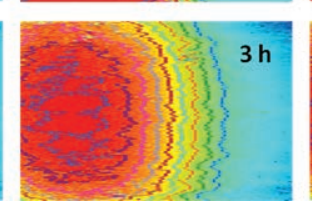

Figure 3. UV absorbance maps showing piroxicam diffusion in $20 \%$ $(\mathrm{w} / \mathrm{w})$ Pluronic $\mathrm{F} 127$ hydrogel after $5 \mathrm{~min}, 0.5 \mathrm{~h}, 1 \mathrm{~h}, 2 \mathrm{~h}, 3 \mathrm{~h}$, and $4 \mathrm{~h}$. Concentration range from $0.2 \mathrm{mM}$ piroxicam (red) to zero (blue). The contour lines represent iso-concentration lines. The rectangular bar shows the image area selected for determination of concentration-distance profiles. Measurement was performed at $355 \mathrm{~nm}$ and $26 \pm 1{ }^{\circ} \mathrm{C}$. Reprinted with permission from ref 66. Copyright 2011 Elsevier. 
lines were caused by swelling of the solid drug substance or solid form change, in situ Raman spectroscopy was applied in a flow-through dissolution cell with similar geometry. The results show that the solid form of the furosemide samples did not change during the dissolution experiments. Thus real-time UV imaging was reported to provide both qualitative and quantitative information about furosemide dissolution behavior in biorelevant media.

Using an almost similar setup, Nielsen et al. (51) investigated the dissolution behavior of amorphous furosemide, crystalline furosemide, amorphous furosemide sodium salt, and crystalline furosemide sodium salt in a biorelevant intestinal medium containing $2.5 \mathrm{mM}$ phosphatidyl choline and $10 \mathrm{mM}$ sodium taurocholate at pH 6.5 (23-25 ${ }^{\circ} \mathrm{C}$ ). The amorphous furosemide forms (acid and salt) were prepared by spray-drying, and the amorphous furosemide salt had high stability during storage at $22^{\circ} \mathrm{C}$ and $33 \%$ relative humidity (stable for 291 days) (53). The rapidly dissolving amorphous and crystalline sodium salts of furosemide lead to absorbance values above the linear range of the calibration curve. Therefore the UV images were not suitable for determining the dissolution rates of these compacts which was instead done by quantification of the concentration of furosemide in the collected effluent (51). For amorphous and crystalline furosemide, good consistency was observed for the dissolution rates determined by furosemide sampling in the effluent and from the UV images as well as dissolution rates determined using a $\mu D I S S$ profiler. This study (51) is likely to represent the first direct comparison between the two microscale dissolution testing methods; additional studies comparing the methods are of interest. The dissolution studies show that the amorphous furosemide salt had the highest dissolution rate followed by the crystalline salt. The amorphous and crystalline furosemide exhibited similar and the lowest dissolution rates. Adjunct solid-form characterization studies by Raman and X-ray powder diffraction indicate that a rapid conversion of the amorphous form to crystalline furosemide negated the higher dissolution rates expected for the more soluble amorphous solid (51). Interestingly, both the amorphous and the crystalline furosemide sodium salt converted to a trihydrate salt during dissolution into the biorelevant medium $(\mathrm{pH} 6.5$ and $23-25^{\circ} \mathrm{C}$ ). Upon dosing to rats, the higher apparent solubility and dissolution rate of the amorphous furosemide sodium salt only translated into a faster $T_{\max }$ as compared with amorphous furosemide and crystalline furosemide. The $A U C$ values were not significantly different among the three solid forms (53).

The dissolution of celecoxib, ketoprofen, naproxen, and sulfathiazole in fasted-state simulated intestinal fluid ( $\mathrm{FaS}$ SIF), pH 6.5, was studied by Niederquell and Kuentz (49). A heterogeneous approach to drug dissolution was taken focusing on the early time points of the experiments. High initial dissolution rates were observed for the four drug substances, but over time, the dissolution rates decreased and a pseudo-equilibrium state was approached. The dissolution rates determined at pseudo-equilibrium conditions were comparable with previously determined dissolution rates for the APIs. Similar to previous UV imaging dissolution studies $(11,36,48)$, significant variability was observed among samples, especially at the early time points. This is related to sample heterogeneity, which was qualitatively assessed by scanning electron microscopy (SEM). Double logarithmic plots of dissolution rate versus time were applied to model heterogeneity effects; fractallike dissolution behavior was observed since the profiles adhere to a power law relation. This interesting study (49) raises an important issue of how heterogeneity-related effects (e.g., sample surface heterogeneity) will affect dissolution experiments performed using miniaturized dissolution testing methods such as the UV imaging system.

The application of UV imaging for determination of intrinsic dissolution rates in plain buffer and biorelevant media appears promising. However, comprehensive studies establishing repeatability and correlation with values determined using traditional dissolution testing apparatus as has been done for the miniaturized rotating disk method (9) would be of huge interest and importance.

\section{Dissolution from Single Crystals and Compacted Nanocrystals}

The dissolution of single lidocaine crystals in stagnant phosphate buffer, pH 7.4 (54), constitutes a most illustrative example highlighting the potential of UV imaging. A single crystal $(\approx 3 \mathrm{~mm})$ of the highly soluble compound lidocaine was mounted in a special flow-through dissolution cell; the cell was filled with the buffer, and the flow was arrested while UV imaging was conducted at 254 $\mathrm{nm}$. The UV images provided spatially and temporally resolved mapping of apparent lidocaine concentrations immediately next to the crystal during dissolution. The absorbance values in the three-dimensional flow cell were averaged over the light path $(3 \mathrm{~mm}$ in the lidocaine study), and thus, should be referred to as apparent concentrations. UV absorbance maps documented natural convection effects due to density gradients forming during lidocaine dissolution (i.e., essentially the occurrence of asymmetric concentration patterns due to concentrated [dense] lidocaine solution falling to the bottom of the quartz cell). Quantitative estimates of the amount of lidocaine dissolved were also obtained from the UV images.

Drug nanocrystals may offer an interesting approach in formulation development of poorly soluble active pharmaceutical ingredients (55-57). Sarnes et al. (58) prepared indomethacin nanocrystals using poloxamer F68 and poloxamer F127 as stabilizers. Wet-milling was applied to produce nanocrystal suspensions, which were subsequently freeze-dried in the presence of lactose to prevent aggregation leading to nanocrystal preparations with particle sizes in the range of 580-950 nm (58). The dissolu- 
tion properties of the poloxamer F68 and poloxamer F127 stabilized nanocrystals were investigated by a traditional flow-through dissolution method (channel flow dissolution method) as well as UV imaging upon the formation of drug nanocrystal compacts. Initial UV imaging-based dissolution experiments on the nanocrystal compacts were performed using the flow-through setup. In the presence of flow, a strong absorbance at $265 \mathrm{~nm}$ in the UV images indicates rapid dissolution of indomethacin from the compact in acetate buffer, $\mathrm{pH} 5.0$. However, at $550 \mathrm{~nm}$, where indomethacin does not absorb light, significant light absorption was detected indicating that nanoparticles escaped the sample surface leading to the scattering of light instead of or in addition to molecularly dissolved indomethacin (58). Van Eerdenbrugh et al. (59) have previously shown that in addition to scattering, submicronsized particulates may affect the UV spectra by absorption of the light. Both effects lead to an apparent increase in absorbance of the particulate-containing solution. It was of interest to investigate the dissolution properties while suppressing the nanocrystal disappearance. This was accomplished by investigating the dissolution under stagnant conditions with the quartz cell filled with $1 \%$ agarose hydrogel/acetate buffer. Application of these dissolution testing conditions to the indomethacin nanocrystal compact abolished absorption at $550 \mathrm{~nm}$, while indomethacin dissolution was apparent with increasing absorbance observed at $265 \mathrm{~nm}$. Subject to these conditions, the nanocrystal compacts exhibited a much higher dissolution rate as compared with compacts containing bulk indomethacin (58). However, in contrast to the flow-through dissolution method, where dissolution rate increased with decreasing particle size of the compacted poloxamerstabilized nanocrystals, significantly different dissolution rates were not observed by UV imaging. Indomethacin concentrations well above the solubility of indomethacin were measured in the agarose gel immediately above the poloxamer stabilized nanocrystal compact surfaces. The authors suggested that this was due to supersaturation (58). However, it may be difficult to rule out (local) solubility enhancing effects of poloxamer F68 and F127 as a contributing factor to the high local indomethacin concentrations observed.

\section{HPMC Swelling}

The swelling behavior of hydroxypropyl methylcellulose (HPMC) compacts in buffer solution was studied by UV imaging under stagnant conditions as well as in the presence of flow using the flow cell shown in Figure 1 (60). Compacts of two viscosity grades of HPMC, $15 \mathrm{CP}$ and 50 $\mathrm{CP}$, were prepared and subjected to real-time visualization of the polymer behavior at $214 \mathrm{~nm}$. Three distinctive phases were observed during the swelling of HPMC: the gel formation, the expansion of the gel into the solution, and steady-state conditions where the polymer concentration is maintained at a critical concentration at a certain distance from the original sample surface. The UV imaging results were related to rheological measurements, and the critical concentration obtained by UV imaging corresponded to the gel point determined by rheological measurements. The higher viscosity grade HPMC had a lower gel point concentration, as expected (60). Furthermore, UV imaging shows that the higher viscosity grade HPMC swelled more rapidly and led to a thicker gel layer, which was more resistant toward the shear forces induced by the flow of the dissolution medium (60). The HPMC may cause a decrease in the light transmitted through the cell by absorbance of light, scattering of the light, or the physical blockage of light by undissolved polymer particles. A practical approach was taken to address this issue by preparing an HPMC calibration curve for relating measured absorbance values to apparent HPMC concentrations. The release of drug from such HPMC compacts remains to be studied by UV imaging. This may be nontrivial since a wavelength at which the drug, but not the polymer, absorbs or scatters light has to be identified and selected.

\section{IN VITRO DRUG RELEASE}

This section covers the attempts to apply UV imaging technology to the investigation of in vitro drug release from pharmaceutical dosage forms, including patches, hydrogels, and lipophilic solutions, using microliter volumes of release media.

\section{Release of Nicotine from Transdermal Patch Samples}

The rate of nicotine release from Nicorette patch samples into phosphate buffer solution, $\mathrm{pH} 7.40$, was studied in a first application of UV imaging (31). Nicorette patches for transdermal application are designed to provide sustained release of nicotine $(\approx 16 \mathrm{~h})$. In the experiments performed, patch samples with a diameter of $2 \mathrm{~mm}$ were cored out of the commercial patches and mounted in the flow cell (Figure 1), where upon the release and diffusion of nicotine was followed. Initially, the placement of a Nicorette patch sample into a stagnant phosphate buffer solution enabled monitoring in real time the formation and development of concentration gradients (concentration-distance profiles) next to the patch surface. Highly variable results were attained when the nicotine release from the patch samples into a continuous flow of buffer was investigated. This was thought to be due to insufficient detector sensitivity for monitoring the slow rate of nicotine release from the patch. However, our current perspective on these very initial experiments is that it was more likely that our sample handling and placement was inadequate. Instead, a flow-stopped-flow procedure was developed, where the flow was stopped for intervals of 3-10 $\mathrm{min}$ followed by a flush $(2.0 \mathrm{~mL} / \mathrm{min})$ period of 1 min (31). The increase in UV absorbance during the time periods where the flow was stopped was used to calculate the amount of released nicotine. Release profiles obtained by UV imaging were compared with those obtained using 
the paddle-over-disk method. Higuchi kinetics $(61,62)$ satisfactorily describes the release profiles obtained by both methods, and the release profiles obtained by UV imaging are in qualitative agreement with the paddle-over-disk release data (31). However, the rate of nicotine release per unit area obtained from UV imaging was significantly higher than the release rate obtained by the paddle-overdisk method for the intact patches. The higher nicotine release rate from the samples investigated by UV imaging was most likely due to an increased release from the edges of the patch samples leading to an increased effective area for drug release (31). Variability in the patch surface area is presumed also to be a contributing source to the relatively high variability with respect to release rates.

\section{Drug Diffusion in and Release from Hydrogels}

Hydrogels are widely used in the biomedical sciences for drug delivery and tissue-engineering purposes (63-65). The polymeric networks imbibing high amounts of water are useful for sustaining and controlling drug release, often via diffusion or erosion mechanisms. Current theoretical models are unable to accurately predict drug diffusion behavior in hydrogels, and experimental methods are needed. UV imaging-based diffusion studies were introduced using the nonionic poly(ethylene oxide)-poly(propylene oxide)-poly(ethylene oxide) (PEO-PPO-PEO) triblock copolymer Pluronic F127 to form the hydrogel, with piroxicam and human serum albumin as diffusants (66). Drug diffusion in and release from Pluronic F127 hydrogels was performed in rectangular parallel piped quartz cells $(7.0 \times 3.0 \times 63 \mathrm{~mm})$ by placing the drug-loaded gel in contact with the release medium (blank hydrogel or aqueous solution). Measurement of the UV absorbance as a function of time provided spatially (2D) and temporally resolved absorbance (concentration) maps of piroxicam at $355 \mathrm{~nm}$ (Figure 3) or human serum albumin at $280 \mathrm{~nm}$ (66). Using Fick's second law, the diffusion coefficients of the low molecular weight substance and the model protein in Pluronic F127 hydrogels at varying polymer concentrations were determined from constructed concentration-distance profiles. As compared with the diffusion coefficients in aqueous solution (phosphate buffer, pH 7.4), the apparent diffusivities of piroxicam and human serum albumin in $20 \%(\mathrm{w} / \mathrm{w}) \mathrm{F} 127$ gels, $\mathrm{pH} 7.4$, were decreased 7.5 and 24 times, respectively. Also the release of piroxicam from F127 hydrogels into phosphate buffer, $\mathrm{pH} 7.4$, was investigated. A magnetic stirring bar was placed in the aqueous solution to attain uniform drug concentrations in the release medium. This experimental setup (66) provided information pertaining to the piroxicam concentrations in the gel phase, the boundary polymer layer, as well as the bulk aqueous phase in real time (Figure 4a). The dissolution (erosion) of the gel was apparent from the UV absorbance maps as a moving gel-buffer front. The amount of piroxicam released (Figure 4b) was calculated both from the gel dis- a
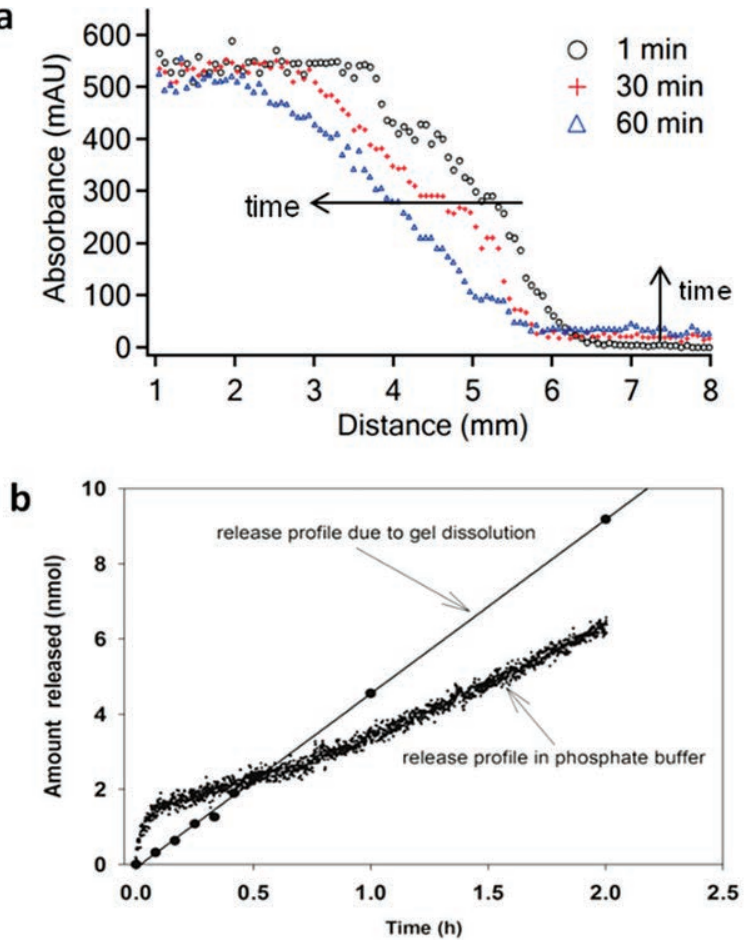

Figure 4. UV imaging of piroxicam release from $30 \%(w / W) F 127$ hydrogels into $67 \mathrm{mM}$ phosphate buffer ( $\mathrm{pH} 7.4$ ) at $355 \mathrm{~nm}$; (a) UV absorbance-distance profiles at different time points; (b) piroxicam release profiles determined both from the gel dissolution and the accumulation of the drug in the bulk release media. Reprinted with permission from ref 66. Copyright 2011 Elsevier.

solution and the accumulation in the bulk release medium (66). These preliminary release experiments suggest that UV imaging-based methods may be helpful in providing a more detailed understanding of the processes and mechanisms of release from hydrogel-based and related delivery systems in a nonintrusive manner. UV imaging may provide a viable alternative to, for instance, fluorescence microscopy (65), nuclear magnetic resonance (NMR) $(65,67)$, Fourier transform infrared (FTIR) imaging $(30,68)$, UV-vis spectroscopy $(69,70)$, and other optical techniques (71) for investigation of diffusion properties. However, these types of studies would benefit from an investigation of the functional spatial resolution of the UV imaging instrumentation since light scattering may be expected due to the presence of a gel.

\section{Release from Lipophilic Solutions into Subcutaneous Tissue Mimic}

Hydrogel-based matrixes have found use as models for soft tissues in relation to drug delivery $(67,72-75)$. It has been proposed that such gels offer a more in vivo-like environment for the study of drug release as compared with in vitro measurement of drug release into agitated bulk liquid phases for parenteral products. However, such gel-based release testing methods have been reported to 
be invasive as well as cumbersome $(72,75)$. UV imaging may offer a noninvasive and less laborious approach to such release studies. The release and transport of piroxicam from a medium-chain triglyceride (MCT) solution into subcutaneous tissue mimicking hydrogels was monitored in real time (76). Measurements were performed in quartz cells $(7.0 \times 3.0 \times 63 \mathrm{~mm})$ by placing the MCT solution in contact with hydrogels consisting of $0.5 \%(\mathrm{w} / \mathrm{v})$ agarose or $25 \%$ (w/v) Pluronic F127 in phosphate buffer, pH 7.4. From the spatially and temporally resolved UV absorbance maps, diffusion coefficients and distribution coefficients were determined using equations derived from Fick's second law (76). The determined MCT-agarose distribution coefficient of 1.4 was identical to the MCT-aqueous distribution coefficient determined using the shakeflask method, whereas that of the MCT-F127 system was four times less. Piroxicam release profiles from MCT solution into the gel matrixes were calculated using the determined transport parameters and the appropriate diffusion model (76). Brandl et al. (65) have suggested the possibility of estimating the drug release profiles from the transport parameters (diffusion coefficient and distribution coefficient) and the appropriate mathematical models instead of using the more time-consuming traditional drug release testing methods, experiments that often span a period of up to several days or weeks. The release from oily vehicles, such as the MCT solutions, has been proposed to be determined, at least partly, by the drug distribution between the oil and the surrounding tissue (77-79). In a subsequent study, Ye et al. (80) investigated the role of piroxicam ionization on the partitioning and release of the drug from MCT solution into hydrogel matrixes by altering $\mathrm{pH}$ in the gel phase. Utilizing a similar UV imaging setup, aqueous and MCT solutions containing piroxicam were injected through a thin capillary into a $0.5 \%(\mathrm{w} / \mathrm{v})$ agarose gel matrix serving as a simple model of subcutaneous tissue. Thus, UV imaging is not limited to the relatively simple models with diffusion into one dimension, although data interpretation is simpler in the case of one-dimensional diffusion, and the relatively small image area of the instrumentation has to be taken into account in the study design. These initial studies indicate that UV imaging in combination with gel matrixes is a promising approach for the characterization of parenterals for injection into soft tissues.

\section{Release from Coated Extrudates}

Gaunø et al. (81) also employed an agarose gel matrix in their studies of 5-aminosalicylic acid release from ethyl cellulose-coated extrudates. The cylindrical extrudates, intended for oral dosing, were composed of the API and povidone and subsequently coated with ethyl cellulose to attain a weight gain of $0.5-2.0 \%(\mathrm{w} / \mathrm{w})$. Single extrudates with a length of approximately $1 \mathrm{~mm}$ were embedded in $0.5 \%(\mathrm{w} / \mathrm{v})$ agarose gel, and the release of 5 -aminosalicylic acid to the surrounding gel matrix was determined by real-time UV imaging. The UV images and corresponding release profiles captured the variability among individual extrudates as well as the effect of ethyl cellulose thickness on drug release (81). In this study, the agarose gel served dual purposes. Attempts to investigate drug release in free solution proved difficult due to the formation of density gradients and natural convective currents, similar to those observed previously (54), which compromised data interpretation (81). The gel efficiently suppressed any density-related effects leading to symmetrical absorbance maps around the extrudate and also acted as a scaffold holding the extrudates in a position completely surrounded by aqueous medium. UV imaging on single extrudates on which the ethyl cellulose coating had been compromised led to asymmetric UV absorbance patterns indicating that UV imaging might be an approach for detection of coating defects (81). The approach utilizing a gel scaffold may provide a means for assessment of drug release from other particulate drug delivery systems.

\section{CONCLUSIONS}

UV imaging facilitates real-time visualization of drug dissolution and release processes as well as subsequent quantification of spatially resolved (2D) drug concentrations in bulk dissolution/release media as well as in the immediate vicinity of an API or a formulation. The technology is well suited for in vitro drug research since many drug substances absorb light in the UV range; the most prominent requirement is transparency of the dissolution medium or sample matrix at the detection wavelength. The possibility of detecting at only a single wavelength at a time may make investigation of complex mixtures and multi component systems challenging. Operation of the commercial instrumentation and data analysis based on Lambert-Beer law is simple and therefore attractive to preformulation and drug formulation labs. Also, the miniaturized or small-scale setup, allowing studies to be performed with only a few milligrams of material, endorses use in the early phases of drug development.

Applications of UV imaging to intrinsic dissolution rate studies are starting to build and results are encouraging. Imaging at the surface of the solid provides visualization of the dissolution events, and especially in combination with solid phase characterization tools, such as Raman spectroscopy, detailed insight into dissolution behavior including solution mediated form changes during dissolution can be attained. A literature review reveals that further work is needed to fully establish the quantitative potential of UV imaging in intrinsic dissolution testing.

Studies on drug diffusion and partitioning in hydrogels demonstrate the versatility of the technology. Characterization of drug release from parenteral formulations may be a fruitful area of application due to the real-time and noninvasive characteristics of UV imaging and the demonstrated compatibility with hydrogels serving as tissue models. 
Interesting pharmaceutical applications of UV imaging have now started to occur in the literature, but a thorough detailed characterization of the instrumentation seems to be missing. Currently there is a lack of data on analytical performance parameters, especially in relation to the functional spatial resolution. However, with the advent of such studies and the continued development of UV imaging technology, we foresee an increased interest in and use of this promising technology.

\section{STATEMENT OF INTEREST}

Jim Lenke is a former employee of Paraytec Ltd., York, UK. The authors declare no conflict of interest. The authors alone are responsible for the content and writing of this paper.

\section{REFERENCES}

1. Amidon, G. L.; Lennernäs, H.; Shah, V. P.; Crison, J. R. A theoretical basis for a biopharmaceutical drug classification: The correlation of in vitro drug product dissolution and in vivo bioavailability. Pharm. Res. 1995, 12 (3), 413-419. DOI: 10.1023/A:1016212804288.

2. Mudie, D. M.; Amidon, G. L.; Amidon, G. E. Physiological parameters for oral delivery and in vitro testing. Mol. Pharm. 2010, 7 (5), 1388-1405. DOI: 10.1021/ mp100149j.

3. Dokoumetzidis, A.; Macheras, P. A century of dissolution research: From Noyes and Whitney to the biopharmaceutics classification system. Int. J. Pharm. 2006, 321 (1-2), 1-11. DOI: 10.1016/j.ijpharm.2006.07.011.

4. McAllister, M. Dynamic dissolution: A step closer to predictive dissolution testing? Mol. Pharm. 2010, 7 (5), 1374-1387. DOI: 10.1021/mp1001203.

5. Dressman, J. B.; Amidon, G. L.; Reppas, C.; Shah, V.P. Dissolution testing as a prognostic tool for oral drug absorption: Immediate release dosage forms. Pharm. Res. 1998, 15 (1), 11-22. DOI: 10.1023/A:1011984216775.

6. Aaltonen, J.; Rades, T. Towards Physico-Relevant Dissolution Testing: The Importance of Solid-State Analysis in Dissolution. Dissolution Technol. 2009, 16 (2), 47-54.

7. Gray, V.; Kelly, G.; Xia, M.; Butler, C.; Thomas, S.; Mayock, S. The science of USP 1 and 2 dissolution: present challenges and future relevance. Pharm. Res. 2009, 26 (6), 1289-1302. DOI: 10.1007/s11095-008-9822-x.

8. Morissette, S. L.; Soukasene, S.; Levinson, D.; Cima, M. J.; Almarsson, Ö. Elucidation of crystal form diversity of the HIV protease inhibitor ritonavir by high-throughput crystallization. Proc. Natl. Acad. Sci. U.S.A. 2003, 100 (5), 2180-2184. DOI: 10.1073/pnas.0437744100.

9. Avdeef, A.; Tsinman, O. Miniaturized rotating disk intrinsic dissolution rate measurement: Effects of buffer capacity in comparisons to traditional Wood's apparatus. Pharm. Res. 2008, 25 (11), 2613-2627. DOI: 10.1007/s11095-008-9679-z.
10. Berger, C. M.; Tsinman, O.; Voloboy, D.; Lipp, D.; Stones, S.; Avdeef, A. Technical Note: Miniaturized Intrinsic Dissolution Rate (Mini-IDR ${ }^{\mathrm{TM}}$ ) Measurement of Griseofulvin and Carbamazapine. Dissolution Technol. 2007, 14 (4), 39-41.

11. Boetker, J. P.; Savolainen, M.; Koradia, V.; Tian, F.; Rades, T.; Müllertz, A.; Cornett, C.; Rantanen, J.; Østergaard, J. Insights into the early dissolution events of amlodipine using UV imaging and Raman spectroscopy. Mol. Pharm. 2011, 8 (4), 1372-1380. DOI: 10.1021/ mp200205z.

12. Persson, A. M.; Baumann, K.; Sundelöf, L.-A.; Lindberg, W.; Sokolowski, A.; Pettersson, C. Design and characterization of a new miniaturized rotating disk equipment for in vitro dissolution rate studies. J. Pharm. Sci. 2008, 97 (8), 3344-3355. DOI: 10.1002/jps.21235.

13. Tsinman, K.; Avdeef, A.; Tsinman, O.; Voloboy, D. Powder dissolution method for estimating rotating disk intrinsic dissolution rates of low solubility. Pharm. Res. 2009, 26 (9), 2093-2100. DOI: 10.1007/s11095-009-9921-3.

14. Gravestock, T.; Box, K.; Frake, E.; Judge, S.; Ruiz, R. The "Gl dissolution" method: a low volume, in vitro apparatus for assessing the dissolution/precipitation behaviour of an active pharmaceutical ingredient under biorelevant conditions. Anal. Methods 2011, 3 (3), 560-567. DOI: 10.1039/c0ay00434k.

15. Aaltonen, J.; Heinänen, P.; Peltonen, L.; Kortejärvi, H.; Tanninen, V. P.; Christiansen, L.; Hirvonen, J.; Yliruusi, J.; Rantanen, J. In situ measurement of solvent-mediated phase transformations during dissolution testing. J. Pharm. Sci. 2006, 95 (12), 2730-2737. DOI: 10.1002/ jps.20725.

16. Greco, K.; Bogner, R. Solution-mediated phase transformations: Significance during dissolution and implications for bioavailability. J. Pharm. Sci. 2012, 101 (9), 2996-3018. DOI: 10.1002/jps.23025.

17. Larsen, C.; Larsen, S. W.; Jensen, H.; Yaghmur, A.; Østergaard, J. Role of in vitro release models in formulation development and quality control of parenteral depots. Expert Opin. Drug Delivery 2009, 6 (12), 12831295. DOI: $10.1517 / 17425240903307431$.

18. Iyer, S. S.; Barr, W. H.; Karnes, H. T. Profiling in vitro drug release from subcutaneous implants: A review of current status and potential implications on drug product development. Biopharm. Drug Dispos. 2006, 27 (4), 157-170. DOI: 10.1002/bdd.493.

19. D’Souza, S. S.; Deluca, P. P. Methods to assess in vitro drug release from injectable polymeric particulate systems. Pharm. Res. 2006, 23 (3), 460-474. DOI: 10.1007/ s11095-005-9397-8.

20. Shen, J.; Burgess, D. J. Accelerated in-vitro release testing methods for extended-release parenteral dosage forms. J. Pharm. Pharmacol. 2012, 64 (7), 986-996. DOI: 10.1111/j.2042-7158.2012.01482.x.

21. Seidlitz, A.; Weitschies, W. In-vitro dissolution methods for controlled release parenterals for controlled release 
parenterals and their applicability to drug-eluting stent testing. J. Pharm. Pharmacol. 2012, 64 (7), 969-985. DOI: 10.1111/j.2042-7158.2011.01439.x.

22. Metz, H.; Mader, K. Benchtop-NMR and MRI-A new analytical tool in drug delivery research. Int. J. Pharm. 2008, 364 (2), 170-175. DOI: 10.1016/j. ijpharm.2008.09.033.

23. Kazarian, S. G.; Van der Weerd, J. Simultaneous FTIR spectroscopic imaging and visible photography to monitor tablet dissolution and drug release. Pharm. Res. 2008, 25 (4), 853-860. DOI: 10.1007/s11095-007-9375-4.

24. Richardson, J. C.; Bowtell, R. W.; Mäder, K.; Melia, C. D. Pharmaceutical applications of magnetic resonance imaging (MRI). Adv. Drug Del. Rev. 2005, 57 (8), 11911209. DOI: 10.1016/j.addr.2005.01.024.

25. Van der Weerd, J.; Kazarian, S. G. Combined approach to FTIR imaging and conventional dissolution tests applied to drug release. J. Controlled Release 2004, 98 (2), 295-305. DOI: 10.1016/j.jconrel.2004.05.007.

26. Zhang, Q.; Gladden, L.; Avalle, P.; Mantle, M. In vitro quantitative ${ }^{1} \mathrm{H}$ and ${ }^{19} \mathrm{~F}$ nuclear magnetic resonance spectroscopy and imaging studies of fluvastatin ${ }^{\mathrm{TM}}$ in Lescol ${ }^{\circledR} \mathrm{XL}$ tablets in a USP-IV dissolution cell. J. Controlled Release 2011, 156 (3), 345-354. DOI: 10.1016/j.jconrel.2011.08.039.

27. Nott, K. P. Magnetic resonance imaging of tablet dissolution. Eur. J. Pharm. Biopharm. 2010, 74 (1), 78-83. DOI: 10.1016/j.ejpb.2009.07.003.

28. Windbergs, M.; Jurna, M.; Offerhaus, H. L.; Herek, J. L.; Kleinebudde, P.; Strachan, C. J. Chemical imaging of oral solid dosage forms and changes upon dissolution using coherent anti-Stokes raman scattering microscopy. Anal. Chem. 2009, 81 (6), 2085-2091. DOl: 10.1021/ ac8020856.

29. Mantle, M. D. NMR and MRI studies of drug delivery systems. Curr. Opin. Colloid Interface Sci. 2013, 18 (3), 214-227. DOI: 10.1016/j.cocis.2013.03.006.

30. Kazarian, S. G.; Ewing, A. V. Applications of Fourier transform infrared spectroscopic imaging to tablet dissolution and drug release. Expert Opin. Drug Delivery 2013, 10 (9), 1207-1221. DOI: 10.1517/17425247.2013.801452.

31. Østergaard, J.; Meng-Lund, E.; Larsen, S. W.; Larsen, C.; Petersson, K.; Lenke, J.; Jensen, H. Real-time UV imaging of nicotine release from transdermal patch. Pharm. Res. 2010, 27 (12), 2614-2623. DOI: 10.1007/s11095010-0257-9.

32. Videometer $A / S_{\bar{T}}$ Home Page. http://videometer.com/ (accessed Feb 4, 2014).

33. Paraytec Ltd. Home Page. http://paraytec.com/ (accessed Feb 4, 2014).

34. Wren, S.; Lenke, J. Pharmaceutical dissolution and UV imaging. Am. Lab. 2011, 43 (2), 33-36.

35. Shah, A. C.; Nelson, K. G. Evaluation of a convective diffusion drug dissolution rate model. J. Pharm. Sci. 1975, 64 (9), 1518-1520. DOI: 10.1002/jps.2600640920.
36. Hulse, W. L.; Gray, J.; Forbes, R. T. A discriminatory intrinsic dissolution study using UV area imaging analysis to gain additional insights into the dissolution behaviour of active pharmaceutical ingredients. Int. J. Pharm. 2012, 434 (1-2), 133-139. DOI: 10.1016/j. ijpharm.2012.05.023.

37. de Smidt, J. H.; Fokkens, J. G.; Grijseels, H.; Crommelin, D. J. A. Dissolution of theophylline monohydrate and anhydrous theophylline in buffer solutions. J. Pharm. Sci. 1986, 75 (5), 497-501. DOI: 10.1002/ jps.2600750515.

38. Shefter, E.; Higuchi, T. Dissolution behavior of crystalline solvated and nonsolvated forms of some pharmaceuticals. J. Pharm. Sci. 1963, 52 (8), 781-791. DOI: 10.1002/jps.2600520815.

39. Boetker, J.; Rades, T.; Rantanen, J.; Hawley, A.; Boyd, B. J. Structural elucidation of rapid solution-mediated phase transitions in pharmaceutical solids using in situ synchroton SAXS/WAXS. Mol. Pharm. 2012, 9 (9), 2787-2791. DOI: 10.1021/mp3003072.

40. Ono, M.; Tozuka, Y.; Oguchi, T.; Yamamoto, K. Effects of dehydration temperatures on moisture absorption and dissolution behavior of theophylline. Chem. Pharm. Bull. 2001, 49 (12), 1526-1530. DOI: 10.1248/ cpb.49.1526.

41. Rodríguez-Hornedo, N.; Lechuga-Ballesteros, D.; Wu, H.-J. Phase transition and heterogeneous/epitaxial nucleation of hydrated and anhydrous theophylline crystals. Int. J. Pharm. 1992, 85 (1-3), 149-162. DOI: 10.1016/0378-5173(92)90144-Q.

42. Qiao, N.; Li, M.; Schlindwein, W.; Malek, N.; Davies, A.; Trappitt, G. Pharmaceutical cocrystals: an overview. Int. J. Pharm. 2011, 419 (1-2), 1-11. DOI: 10.1016/j. ijpharm.2011.07.037.

43. Thakuria, R.; Delori, A.; Jones, W.; Lipert, M. P.; Roy, L.; Rodríguez-Hornedo, N. Pharmaceutical cocrystals and poorly soluble drugs. Int. J. Pharm. 2013, 453 (1), 101125. DOI: 10.1016/j.ijpharm.2012.10.043.

44. Elder, D. P.; Holm, R.; de Diego, H. L. Use of pharmaceutical salts and cocrystals to address the issue of poor solubility. Int. J. Pharm. 2013, 453 (1), 88-100. DOI: 10.1016/j.ijpharm.2012.11.028.

45. Schultheiss, N.; Newman, A. Pharmaceutical cocrystals and their physicochemical properties. Crystal Growth Des. 2009, 9 (6), 2950-2967. DOI: 10.1021/ cg900129f.

46. Li, M.; Qiao, N.; Wang, K. Influence of sodium lauryl sulfate and Tween 80 on carbamazepine-nicotinamide cocrystal solubility and dissolution behaviour. Pharmaceutics 2013, 5 (4), 508-524. DOI: 10.3390/pharmaceutics5040508.

47. Qiao, N.; Wang, K.; Schlindwein, W.; Davies, A.; Li, M. In situ monitoring of carbamazepine-nicotinamide cocrystal intrinsic dissolution behaviour. Eur. J. Pharm. Biopharm. 2013, 83 (3), 415-426. DOI: 10.1016/j. ejpb.2012.10.005. 
48. Gordon, S.; Naelapää, K.; Rantanen, J.; Selen, A.; Müllertz, A.; Østergaard, J. Real-time dissolution behavior of furosemide in biorelevant media as determined by UV imaging. Pharm. Dev. Technol. 2013, 18 (6), 1407-1416. DOI: 10.3109/10837450.2012.737808.

49. Niederquell, A.; Kuentz, M. Biorelevant dissolution of poorly soluble weak acids studied by UV imaging reveals ranges of fractal-like kinetics. Int. J. Pharm. 2014, 463 (1), 38-49. DOI: 10.1016/j. ijpharm.2013.12.049.

50. Boetker, J. P.; Rantanen, J.; Rades, T.; Müllertz, A.; Østergaard, J.; Jensen, H. A new approach to dissolution testing by UV imaging and finite element simulation. Pharm. Res. 2013, 30 (5), 1328-1337. DOI: 10.1007/s11095-013-0972-0.

51. Nielsen, L. H.; Gordon, S.; Pajander, J. P.; Østergaard, J.; Rades, T.; Müllertz, A. Biorelevant characterization of amorphous furosemide salt exhibits conversion to a furosemide hydrate during dissolution. Int. J. Pharm. 2013, 457 (1), 14-24. DOI: 10.1016/j. ijpharm.2013.08.029.

52. Østergaard, J.; Wu, J. X.; Naelapää, K.; Boetker, J. P.; Jensen, H.; Rantanen, J. Simultaneous UV Imaging and Raman Spectroscopy for Measurement of SolventMediated Phase Transformations During Dissolution Testing. J. Pharm. Sci. 2014, 103 (4), 1149-1156. DOI 10.1002/jps.23883.

53. Nielsen, L. H.; Gordon, S.; Holm, R.; Selen, A.; Rades, T.; Müllertz, A. Preparation of an amorphous sodium furosemide salt improves solubility and dissolution rate and leads to a faster $\mathrm{T}_{\max }$ after oral dosing to rats. Eur. J. Pharm. Biopharm. 2013, 85 (3), 942-951. DOI: 10.1016/j. ejpb.2013.09.002.

54. Østergaard, J.; Ye, F.; Rantanen, J.; Yaghmur, A.; Larsen, S. W.; Larsen, C.; Jensen, H. Monitoring lidocaine singlecrystal dissolution by ultraviolet imaging. J. Pharm. Sci. 2011, 100 (8), 3405-3410. DOI: 10.1002/jps.22532.

55. Merisko-Liversidge, E.; Liversidge, G. G. Nanosizing for oral and parenteral drug delivery: A perspective on formulating poorly water soluble compounds using wet media milling technology. Adv. Drug Del. Rev. 2011, 63 (6), 427-440. DOI: 10.1016/j.addr.2010.12.007.

56. Möschwitzer, J. P. Drug nanocrystals in the commercial pharmaceutical development process. Int. J. Pharm. 2013, 453 (1), 142-156. DOI: 10.1016/j. ijpharm.2012.09.034.

57. Keck, C. M.; Müller, R. H. Drug nanocrystals of poorly soluble drugs produced by high pressure homogenisation. Eur. J. Pharm. Biopharm. 2006, 62 (1), 3-16. DOI: 10.1016/j.ejpb.2005.05.009.

58. Sarnes, A.; Østergaard, J.; Jensen, S. S.; Aaltonen, J.; Rantanen, J.; Hirvonen, J.; Peltonen, L. Dissolution study of nanocrystal powders of a poorly soluble drug by UV imaging and channel flow methods. Eur. J. Pharm. Sci. 2013, 50 (3-4), 511-519. DOI: 10.1016/j. ejps.2013.08.030.
59. Van Eerdenbrugh, B.; Alonzo, D. E.; Taylor, L. S. Influence of particle size on the ultraviolet spectrum of particulate-containing solutions: Implications for in-situ concentration monitoring using UV/Vis Fiberoptic probes. Pharm. Res. 2011, 28 (7), 1643-1652. DOI: 10.1007/s11095-011-0399-4.

60. Pajander, J.; Baldursdottir, S.; Rantanen, J.; Østergaard, J. Behaviour of HPMC compacts investigated using UV-imaging. Int. J. Pharm. 2012, 427 (2), 345-353. DOI: 10.1016/j.ijpharm.2012.02.034.

61. Higuchi, T. Physical chemical analysis of percutaneous absorption process from creams and ointments. J. Soc. Cosmet. Chem. 1960, 11 (2), 85-97.

62. Higuchi, W. I. Analysis of data on the medicament release from ointments. J. Pharm. Sci. 1962, 51 (8), 802-804. DOI: 10.1002/jps.2600510825.

63. Van Vlierberghe, S.; Dubruel, P.; Schacht, E. Biopolymerbased hydrogels as scaffolds for tissue engineering applications: a review. Biomacromolecules 2011, 12 (5), 1387-1408. DOI: 10.1021/bm200083n.

64. Jung, H. H.; Park, K.; Han, D. K. Preparation of TGF-beta 1-conjugated biodegradable Pluronic F127 hydrogel and its application with adipose-derived stem cells. J. Controlled Release 2010, 147 (1), 84-91. DOI: 10.1016/j. jconrel.2010.06.020.

65. Brandl, F.; Kastner, F.; Gschwind, R. M.; Blunk, T.; Teßmar, J.; Göpferich, A. Hydogel-based drug delivery systems: Comparison of drug diffusivity and release kinetics. J. Controlled Release 2010, 142 (2), 221-228. DOI: 10.1016/j.jconrel.2009.10.030.

66. Ye, F.; Yaghmur, A.; Jensen, H.; Larsen, S. W.; Larsen, C.; Østergaard, J. Real-time UV imaging of drug diffusion and release from Pluronic F127 hydrogels. Eur. J. Pharm. Sci. 2011, 43 (4), 236-243. DOI: 10.1016/j. ejps.2011.04.015.

67. Klose, D.; Azaroual, N.; Siepmann, F.; Vermeersch, G.; Siepmann, J. Towards more realistic in vitro release measurement techniques for biodegradable microparticles. Pharm. Res. 2009, 26 (3), 691-699. DOI: 10.1007/ s11095-008-9747-4.

68. Rafferty, D. W.; Koenig, J. L. FTIR imaging for the characterization of controlled-release drug delivery applications. J. Controlled Release 2002, 83 (1), 29-39. DOI: 10.1016/S0168-3659(02)00135-9.

69. Anderson, B. C.; Pandit, N. K.; Mallapragada, S. K. Understanding drug release from poly(ethylene oxide)$b$ poly(propylene oxide)-b-poly(ethylene oxide) gels. J. Controlled Release 2001, 70 (1-2), 157-167. DOI: 10.1016/S0168-3659(00)00341-2.

70. Dunmire, E. N.; Plenys, A. M.; Katz, D. F. Spectrophometric analysis of molecular transport in gels. J. Controlled Release 1999, 57 (2), 127-144. DOI: 10.1016/S0168-3659(98)00111-4.

71. Ambrosini, D.; Paolett, D.; Rashidnia, N. Overview of diffusion measurements by optical techniques. Opt. 
Lasers Eng. 2008, 46 (12), 852-864. DOI: 10.1016/j.optlaseng.2008.06.008.

72. Allababidi, S.; Shah, J. C. Kinetics and mechanism of release from glyceryl monostearate-based implants: Evaluation of release in gel simulating in vivo implantation. J. Pharm. Sci. 1998, 87 (6), 738-744. DOI: $10.1021 /$ js9703986.

73. Gietz, U.; Arvinte, T.; Mader, E.; Oroszlan, P.; Merkle, H. P. Sustained release of injectable zinc-recombinant hirudin suspensions: development and validation of in vitro release model. Eur. J. Pharm. Biopharm. 1998, 45 (3), 259-264. DOI: 10.1016/ S0939-6411(98)00008-3.

74. Peschka, R.; Dennehy, C.; Szoka Jr, F. C. A simple in vitro model to study the release kinetics of liposome encapsulated material. J. Controlled Release 1998, 56 (1-3), 41-51. DOI: 10.1016/S0168-3659(98)00067-4.

75. Hoang Thi, T. H.; Chai, F.; Leprêtre, S.; Blanchemain, N.; Martel, B.; Siepmann, F.; Hildebrand, H. F.; Siepmann, J.; Flament, M. P. Bone implants modified with cyclodextrin: Study of drug release in bulk fluid and into agarose gel. Int. J. Pharm. 2010, 400 (1-2), 74-85. DOI: 10.1016/j.ijpharm.2010.08.035.

76. Ye, F.; Larsen, S. W.; Yaghmur, A.; Jensen, H.; Larsen, C.; Østergaard, J. Real-time UV imaging of piroxicam diffusion and distribution from oil solutions into gels mimicking the subcutaneous matrix. Eur. J. Pharm. Sci. 2012, 46 (1-2), 72-78. DOI: 10.1016/j.ejps.2012.02.011.

77. Zuidema, J.; Kadir, F.; Titulaer, H. A. C.; Oussoren, C. Release and absorption rates of intramuscularly and subcutaneously injected pharmaceuticals (II). Int. J. Pharm. 1994, 105 (3), 189-207. DOI: 10.1016/03785173(94)90103-1.

78. Hirano, K.; Ichihashi, T.; Yamada, H. Studies on the absorption of practically water-insoluble drugs following injection. V. Subcutaneous absorption in rats from solutions in water immiscible oils. J. Pharm. Sci. 1982, 71 (5), 495-500. DOI: 10.1002/jps.2600710505.

79. Larsen, S. W.; Larsen, C. Critical factors influencing the in vivo performance of long-acting lipophilic solutionsimpact on in vitro release method design. AAPS J. 2009, 11 (4), 762-770. DOI: 10.1208/s12248-009-9153-9.

80. Ye, F.; Larsen, S. W.; Yaghmur, A.; Jensen, H.; Larsen, C.; Østergaard, J. Drug release into hydrogel-based subcutaneous surrogates by UV imaging. J. Pharm. Biomed. Anal. 2012, 71, 27-34. DOI: 10.1016/j.jpba.2012.07.024.

81. Gaunø, M. H.; Vilhelmsen, T.; Larsen, C. C.; Boetker, J. P.; Wittendorf, J.; Rantanen, J.; Østergaard, J. Realtime in vitro dissolution of 5-aminosalicylic acid from single ethyl cellulose coated extrudates studied by UV imaging. J. Pharm. Biomed. Anal. 2013, 83, 49-56. DOI: 10.1016/j.jpba.2013.04.028. 